\title{
Revisão dos aspectos botânicos, químicos e farmacológicos de Adenium obesum (Forssk.) Roem. \& Schult
}

Review of the botanical, chemical and pharmacological aspects of Adenium obesum (Forssk.) Roem. \& Schult

Revisión de los aspectos botánicos, químicos y farmacológicos de Adenium obesum (Forssk.) Roem. \& Schult

\section{Resumo}

Devido as suas propriedades terapêuticas, as plantas são bastante utilizadas pela população para fins medicinais e para estudos científicos a respeito dos constituintes químicos e das ações farmacológicas. A exemplo, pode-se citar a espécie Adenium obesum (Forssk.) Roem. \& Schult. pertencente à família Apocynaceae e popularmente conhecida como rosa do deserto. Na medicina tradicional essa espécie é empregada para tratar diferentes doenças venéreas como a gonorreia e para tratar feridas, infecções, rinite, entre outras afecções. Devido a isso, essa narrativa teve como objetivo descrever os aspectos botânicos, químicos e farmacológicos da espécie. Desse modo, observou-se que a variedade dos constituintes químicos de A. obesum engloba a presença de glicosídeos cardíacos, com aproximadamente 50 fitoquímicos pertencentes à classe dos cardenolídeos, pregnanos, triterpenos e flavonoides. Estes, estão relacionados as atividades biológicas e pode-se constatar a atividades anticâncer, antiviral, antibacteriana, tripanocida, acaricida, moluscicida, antioxidante e pesticida. Portanto, esse compilado de informações de A. obesum mostra a grande importância dessa espécie. Entretanto, torna-se necessário mais pesquisas acerca dos componentes fitoquímicos e atividades biológicas realizadas com diferentes partes da espécie, uma vez que tais componentes podem apresentar favorável potencialidade na incorporação de futuros produtos farmacêuticos.

Palavras-chave: Apocynaceae; Rosa do deserto; Fitoterapia; Botânica; Fitoquímica; Farmacologia.

\begin{abstract}
Due to their therapeutic properties, plants are widely used by the population for medicinal purposes and for scientific studies regarding chemical constituents and pharmacological actions. As an example, we can mention the species Adenium obesum (Forssk.) Roem. \& Schult. belonging to the Apocynaceae family and popularly known as desert rose. In traditional medicine this species is used to treat different venereal diseases such as gonorrhea and to treat wounds, infections, rhinitis, among other conditions. Because of this, this narrative aimed to describe the botanical, chemical and pharmacological aspects of the species. Thus, it was observed that the variety of chemical constituents of A. obesum includes the presence of cardiac glycosides, with approximately 50 phytochemicals belonging to the class of cardenolides, pregnanes, triterpenes and flavonoids. These are related to biological activities and can be seen as anticancer, antiviral, antibacterial, trypanocidal, acaricidal, molluscicidal, antioxidant and pesticidal activities. Therefore, this compilation of information on A. obesum shows the great importance of this species. However, further research is needed on the phytochemical components and biological activities performed with different parts of the species, since such components may have favorable potential in the incorporation of future pharmaceutical products.

Keywords: Apocynaceae; Desert rose; Phytotherapy; Botany; Phytochemistry; Pharmacology.
\end{abstract}

\section{Resumen}

Debido a sus propiedades terapéuticas, las plantas son ampliamente utilizadas por la población con fines medicinales y para estudios científicos sobre componentes químicos y acciones farmacológicas. Como ejemplo, podemos 
mencionar la especie Adenium obesum (Forssk.) Roem. \& Schult. perteneciente a la familia de las Apocynaceae y popularmente conocida como rosa del desierto. En la medicina tradicional esta especie se utiliza para tratar diferentes enfermedades venéreas como la gonorrea y para tratar heridas, infecciones, rinitis, entre otras afecciones. Por eso, esta narrativa tuvo como objetivo describir los aspectos botánicos, químicos y farmacológicos de la especie. Así, se observó que la variedad de constituyentes químicos de A. obesum incluye la presencia de glucósidos cardíacos, con aproximadamente 50 fitoquímicos pertenecientes a la clase de cardenólidos, pregnanos, triterpenos y flavonoides. Estos están relacionados con actividades biológicas y pueden verse como actividades anticancerígenas, antivirales, antibacterianas, tripanocidas, acaricidas, molusquicidas, antioxidantes y plaguicidas. Por tanto, esta recopilación de información sobre $A$. obesum muestra la gran importancia de esta especie. Sin embargo, se necesita más investigación sobre los componentes fitoquímicos y las actividades biológicas realizadas con diferentes partes de la especie, ya que dichos componentes pueden tener un potencial favorable para la incorporación de futuros productos farmacéuticos.

Palabras clave: Apocynaceae; Rosa del desierto; Fitoterapia; Botánica; Fitoquímica; Farmacología.

\section{Introdução}

As espécies vegetais são amplamente utilizadas no mundo e trazem consigo propriedades intrínsecas e únicas. Sendo assim, tornou-se possível desde a antiguidade perceber que para cada mal visto como doença, compostos derivados das plantas proporcionavam a cura (Fabricant \& Farnsworth, 2001). Ainda na história da humanidade, as plantas sempre foram consideradas ótimas opções de tratamento por apresentarem baixo custo e relativamente poucos efeitos colaterais quando comparados com a medicina alopática ou convencional (Springfield et al., 2005).

Vale destacar que plantas pertencentes a família Apocynaceae apresentam grande importância econômica, seja para ornamentação e/ou medicinal. A família é cosmopolita, estando distribuída pelas zonas tropicais e subtropicais do mundo (Hossain et al, 2013; Akhtar et al, 2017).

Dentre as espécies vegetais da família Apocynaceae, encontra-se Adenium obesum (Forssk.) Roem. \& Schult mundialmente conhecida como rosa do deserto e utilizada tradicionalmente como medicamento para tratar diferentes doenças venéreas como a gonorreia, feridas, infecções, rinite (Paul et al., 2015). O líquido isolado da raiz é utilizado para incorporar loções que tratam doenças de pele assim como para pediculose (Akharaiyi, 2011; Tijjani et al., 2011). O látex é utilizado no tratamento de cárie dentária e feridas (Hossain et al, 2013; Akhtar et al, 2017). É também utilizada como pesticida (Versiani et al., 2014), veneno de flecha para caça na África (Oyen, 2008).

De acordo com a literatura os compostos identificados da A. obesum incluem os glicosídeos cardíacos (cardenolídeos), pregnanos, triterpenos, flavonoides e cetildigitoxigenina (Versiani et al., 2014). Estes, estão relacionados as atividades biológicas e pode-se constatar a atividades anticâncer, antiviral, antibacteriana, tripanocida, acaricida, moluscicida, antioxidante e pesticida (Paul et al., 2015).

No Brasil o uso da rosa do deserto é comumente ornamental devido ao ambiente apropriado para cultivo e possui alto valor econômico (Silveira et al., 2016). Entretanto, alguns estudos etnobotânicos destacam a utilização dessa espécie para diferentes fins, bem como para tratar diferentes afecções em comunidades tradicionais (Paul et al., 2015; Akharaiyi, 2011; Tijjani et al., 2011), porém, a relevância desta espécie ainda não foi devidamente estudada, especialmente por apresentar ampla distribuição e ser popularmente empregada na cura de enfermidades. Devido a isso, este estudo apresenta uma revisão dos aspectos botânicos, químicos e farmacológicos de Adenium obesum (Forssk.) Roem. \& Schult.

\section{Metodologia}

Este estudo é uma revisão narrativa de literatura, na qual busca-se captar, reconhecer e sintetizar estudos primários, teóricos e empíricos, possibilitando assim a síntese completa da produção do conhecimento acerca de um assunto ou tema (Souza et al., 2008; Silva \& Silva, 2018). A revisão foi realizada em quatro etapas. Na primeira etapa ocorreu a definição do tema e da questão norteadora: Adenium obesum (Forssk.) Roem. \& Schult foi amplamente estudada em estudos químicos e 
farmacológicos? Na segunda etapa definiram-se os critérios de inclusão e de exclusão. Os critérios de inclusão foram artigos na versão on-line e em bases de dados de acesso gratuito e privado, nacionais e internacionais (português, inglês e espanhol), sem espaço temporal delimitado. Foram excluídas publicações que, após a leitura minuciosa, não estavam relacionadas ao tema e objetivo e também publicações repetidas. A terceira fase abordou a seleção das referências. Realizou-se uma busca bibliográfica criteriosa em artigos científicos disponíveis em bases de dados do Scielo, Science Direct e Google Scholar, conhecidas por abordagens de pesquisas baseadas em evidências científicas. Os descritores considerados foram A. obesum, constituintes químicos, fitoquímica, química, atividade farmacológica e atividade biológica. Na quarta etapa foram avaliados os artigos selecionados.

\section{Aspectos Botânicos de Adenium obesum (Forssk.) Roem. \& Schult}

A. obesum pode ser encontrada como planta selvagem em países como o Iêmen, Quênia, Sudão, Senegal, Etiópia, Arábia Saudita e Omã (Limones et al., 2018). No Brasil, a espécies é bastante difundida como planta ornamental, estando presente em diferentes estados e biomas (Gonçalves \& Pasa, 2015).

É uma espécie arbustiva e suculenta do tipo caudiciforme (Figura1). Sua adaptação foi essencial para sobreviver em lugares muito quentes onde a escassez de água é bastante frequente; portanto, ela desenvolveu mecanismos para acumular água. Por esse motivo, apresenta um espessamento na parte inferior do caule conhecido como caudex, cujo o objetivo é armazenar água. Devido a essa característica peculiar, recebe o nome de A. obesum, que se refere à espessura do caule. Além de apresentar o caudex, que lhe confere uma característica especial, também possui flores muito vistosas (Varella et al., 2015; Limones et al., 2018) e é uma planta de fácil cultivo, pois tolera diversos tipos de solos, altas temperaturas e baixa necessidade de água (Orozco \& Gonzales, 2021).

Sua floração acontece comumente na primavera, no entanto, pode ser observada também no outono e no verão. As flores tem formato tubular, o seu desenvolvimento conta com a presença abundante de luz e normalmente são multicoloridas, mas sem cheiro (Romahn, 2012). A fruta é folícular e na maturidade se divide para liberar sementes dispersas pelo vento (Paul et al., 2015). Ainda, há a presença da seiva leitosa constituída por compostos químicos tóxicos irritantes à pele (Hossain, 2018).

Figura 1 - Espécie Adenium obesum(Forssk.) Roem. \&Schult.

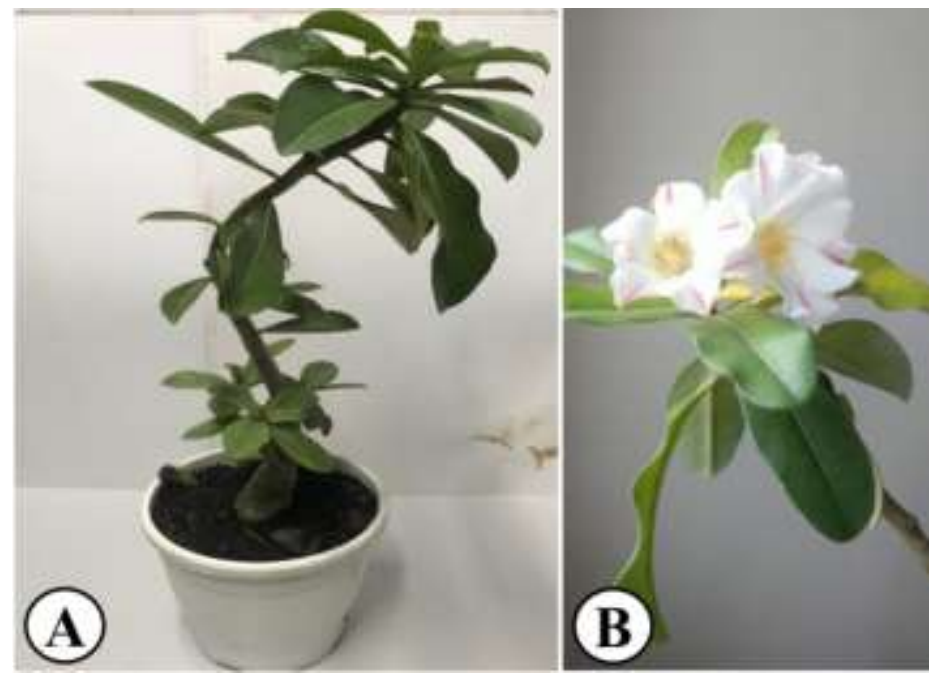

A: Hábito B: arranjo floral. Fonte: Autores. 
A propagação de A. obesum ocorre por sementes e a formação das sementes por autopolinização ou polinização cruzada (Colombo et al. 2015). A espécie em questão pode chegar até quatro metros. No estádio inicial da espécie, a casca tem cor amarelada e com o passar dos dias apresenta-se com um tom marrom a acinzentado (Paul et al., 2015).

As folhas apresentam formato oval e em espiral e estão agrupadas na extremidade do ramo (Paul et al., 2015). Suas folhas são de coloração verde escuro e em condições ideais apresentar um aspecto brilhante (Romahn, 2012). Vale destacar que a espécie quando cultivada na sombra, pode se tornar mais suscetível a doenças. Além disso, deve-se ter cuidado com a drenagem de água, caso isso não ocorra há o apodrecimento das raízes e caules, resultando em um reduzido crescimento ou morte da planta (Silveira et al. 2016).

\section{Constituintes Químicos de Adenium obesum (Forssk.) Roem. \& Schult}

Adenium obesum (Forssk.) Roem. \& Schult apresenta alto valor ornamental. É parte da família Apocynaceae, a qual apresenta grande variabilidade em número e características, segundo Bhadane et al. (2018), apresenta uma rica fonte de espécies fitoquímicas como glicosídeos e possui grande potencial para incorporarem produtos farmacêuticos (Paul et al., 2015).

A variedade de constituintes químicos engloba a presença de glicosídeos cardíacos, com aproximadamente 50 fitoquímicos pertencentes à classe dos cardenolídeos, pregnanos, triterpenos e flavonoides (Versiani et al., 2014). Akhtar et al. (2017), em seu estudo fitoquímico da casca de A. obesum, mostraram que a planta selecionada apresentou diferentes grupos de compostos químicos como por exemplo flavonoides, flavonoides prenilados, terpenóides e pregnanos. Quando isolados, esses compostos químicos se mostraram biologicamente ativos com atividade anticâncer, antiviral, antibacteriana, tripanocida, acaricida, moluscicida, atividade antioxidante e piscicida (Paul et al., 2015).

Destaca-se que os metabólitos secundários são compostos naturais que tem como objetivo principal a proteção contra estresses abióticos e bióticos, além disso, possuem valores nutricionais e farmacológicos que apresentam grande importância na nutrição humana e aditivos aromáticos, corantes e incorporado em formulações farmacêuticas (Borges \& Amorim, 2020), apresentando duas funções adicionais, uma servindo de modelo para a estrutura e síntese de drogas inteiramente novas, e a outra servindo de fonte de material para semi-síntese de agentes medicinais (Kinghorn \& Seo, 1996).

\section{Atividade Farmacológica e Atividade Biológica de Adenium obesum (Forssk.) Roem. \& Schult}

Os constituintes químicos de A. obesum relacionam-se com atividades biológicas comprovadas, tais como, para feridas, doenças de pele, dores nas articulações, dores de cabeça, dores musculares, piolhos, contra doenças sexualmente transmissíveis (gonorreia) e rinite (Paul et al., 2015). Ainda, foi relatado anteriormente que A. obesum tem atividade antiinfluenza contra vírus (Kiyohara et al., 2012), antimicrobiano (Hossain et al., 2017) e atividade citotóxica (Almehdar et al., 2012).

O extrato aquoso da casca triturada da A. obesum de Oman é utilizado para tratar luxações ósseas, reumatismo, entorses, paralisia, inchaços, feridas e infecções de pele (Mclaughlin \& Garofalo, 2002). A decocção da raiz da A. obesum da Somália é utilizada para tratar rinite (Akharaiyi, 2011; Tijjani et al., 2011). O extrato aquoso do caule e da casca esmagada da espécie da Arábia Saudita e Iêmen trata feridas desde a década de 1990 (Dimmitt \& Hanson, 1991). O caule de A. obesum do Quênia triturado, em pó, atua contra parasitas de pele de camelos e gados (Igbinosa et al., 2009). A seguir, descreve-se as principais atividades biológicas de A. obesum comprovadas em estudos científicos: 


\section{- Atividade anticâncer}

Várias moléculas bioativas de A. obesum foram identificadas com o objetivo de prevenir, bem como tratar o câncer. O extrato etanólico da parte aérea da espécie exibiu propriedade citotóxica contra carcinoma epidermóide da nasofaringe de seres humanos. Moléculas bioativas no extrato foram identificadas como honghelosídeo A, honghelina, cardenolídeos somalina, e 16-acetilstrospesida, e o flavonol 3,3'-bis (O-metil) quercetina (Hoffmann \& Cole, 1977).

$\mathrm{Na}$ literatura, foram encontrados dois pregnanos que possuem propriedade citotóxica contra células P388 / S de leucemia murina. Esses, foram isolados a partir de folhas de A. obesum (Nakamura et al., 2000). Almehdar et al. (2012) relataram que extrato metanólico de A. obesum exibiu potencial atividade citotóxica contra três linhagens de células cancerígenas humanas, como câncer de mama (MCF7), câncer de colo do útero (HELA) e carcinoma hepatocelular (HEPG2).

\section{- Atividade imunomoduladora}

Diferentes estudos realizados anteriormente trazem a A. obesum como uma planta que pode oferecer a melhoria do sistema imune. Extratos etanólicos concentrados levaram a um aumento na contagem dos glóbulos brancos, especialmente os linfócitos (Abalaka et al., 2012; Versiani et al., 2014). Ainda, estudos sugerem que a presença de metabólitos secundários com atividade antioxidante de A. obesum preveniu o dano oxidativo e combateu a toxicidade induzida pela própria espécie (Pengelly, 2021; Ramawat \& Merillon, 2010), conotando preliminarmente essa espécie como segura para consumo oral, já que o estudo foi realizado em ratos Wistar, necessitando de outros estudos de toxicidade em ensaios clínicos para a efetiva comprovação (Abalaka et al., 2012)

\section{- Atividade antiviral}

Extratos etanólicos contendo glicosídeo antocianina e glicosídeo cardiotônico, ambos esteroides, de A. obesum apresentaram atividade contra o vírus da influenza (H1N1) (Kiyohara et al., 2012). O composto ativo isolado, responsável pela redução do título do vírus, foi um glicosídeo cardiotônico identificado como oleandrigenina- $\beta$-D glucosil $(1 \rightarrow 4)$ - $\beta$-Ddigitalose (Hossain et al., 2014; Nagai et al., 1995; Zu et al., 2012) A atividade antiviral do extrato de A. obesum foi comprovada utilizando células renais caninas Madin-Darby (MDCK) infectadas com o vírus (Kiyohara et al., 2012).

\section{- Atividade Antibacteriana}

Estudos evidenciaram a atividade antibacteriana de A. obesum presentes em várias partes da espécie contra diferentes microrganismos. O extrato aquoso da casca do caule apresentou satisfatória atividade contra Escherichia coli, Proteus mirabilis, Pseudomonas aeruginosa e Staphylococcus aureus (Adamu et al., 2005). O extrato metanólico das folhas apresentou atividade contra Bacillus amyloliquefaciens, E. coli, P. aeruginosa e S. aureus. E. coli, Proteus vulgaris, Pseudomona saeruginosa e S. aureus foram sensíveis ao extrato metanólico oriundo do tronco (Hossain et al., 2014; Versiani et al., 2014).

\section{- Atividade Tripanocida}

A atividade tripanocida foi evidenciada contra Trypanosoma brucei através do uso de extrato metanólico da casca do caule de A. obesum. O tempo de exposição foi de 1 hora, culminando em $50 \%$ da motilidade de $T$. brucei in vitro a $4 \mathrm{mg} / \mathrm{mL}$ (Atawodi, 2005). Além disso, relatórios de outro estudo sugeriram que diferentes extratos vegetais com éter de petróleo, com clorofórmio, com metanol e frações aquosas mostraram inibição desse parasita T. brucei. O composto ativo identificado foi a botulina, que é um triterpeno, a qual apresentou atividade antiparasitária (Ibrahim et al., 2014; Ramawat \& Merillon, 2010). 


\section{- Atividade Acaricida e Larvicida}

Segundo Mgbojikwe e Okoye (2001), o extrato aquoso de casca de caule de A. obesum mostrou atividade acaricida eficaz contra os carrapatos Boophilus e Amblyomma.

A atividade larvicida com potencial contra larvas do mosquito Aedes aegypti, vetor da febre amarela e doenças da febre hemorrágica da dengue, foi demonstrada através do extrato feito com o caule de A. obesum e Diclorometano (Cepleanu, 1994).

\section{Considerações Finais}

Este compilado dos aspectos botânicos, químicos e farmacológicos de Adenium obesum (Forssk.) Roem. \& Schult mostra a grande importância dessa espécie. Entretanto, apesar dos compostos químicos produzidos e do potencial medicinal dessa espécie já mencionados na literatura, torna-se importante garantir que a utilização de seus componentes não cause toxicidade para os humanos e outros animais, sendo imprescindível a investigação minuciosa em diferentes estudos para a utilização segura desse recurso vegetal.

Portanto, considerando a importância de A. obesum como fonte de compostos com atividades farmacológicas, observa-se a potencialidade dessa espécie e a consequente necessidade de estudos voltados para uma melhor descrição química e biológica.

\section{Referências}

Abalaka, S. E., Fatihu, M. Y., Doguwar, N., Ibrahim, G., \& Ambali, S. F. (2012). Hepatotoxicity of ethanol extract of Adenium obesum stem bark in Wistar rats. British Journal of Pharmaceutical Research, 4 (9), 1041-1052. https://doi.org/10.9734/BJPR/2014/7667

Adamu, H. M., Abayeh, O. J., Agho, M. O, Abdullahi A. L., Uba, A., Dukku, H. U., \& Wufem, B. M. (2005). An ethnobotanical survey of Bauchi State herbal plants and their antimicrobial activity. Journal of Ethnopharmacology, 99 (1), 1-4. https://doi.org/10.1016/j.jep.2004.12.025

Akharaiyi, F. C. (2011). Antibacterial, phytochemical and antioxidant activities of Datura metel. International Journal of PharmTech Research, 3 (1), 478483. https://sphinxsai.com/Vol.3No.1/pharm_jan-mar11/pdf/JM11(PT=79)\%20pp\%20478-483.pdf

Akhtar, M. S., Hossain, M. A., \& Said, S. A. (2017). Isolation and characterization of antimicrobial compound from the stem-bark of the traditionally used medicinal plant Adenium obesum. Journal of Traditional and Complementary Medicine, 7 (3), 296-300. https://doi.org/10.1016/j.jtcme.2016.08.003

Almehdar, H., Abdallah, H. M., Osman, A-B. M., \& Sattar, E. A. A. (2012). In vitro cytotoxic screening of selected Saudi medicinal plants. Journal of natural medicines, 66 (2), 406-412. https://doi.org/10.1007/s11418-011-0589-8

Atawodi, S. E. (2005). Comparative in vitro trypanocidal activities of petroleum ether, chloroform, methanol and aqueous extracts of some Nigerian savannah plants. African journal of Biotechnology, 4 (2), 177-182. https://www.ajol.info/index.php/ajb/article/view/15075

Bhadane, B. S., Patil, M. P., Maheshwari, V. L., \& Patil, R. H. (2018). Ethnopharmacology, phytochemistry, and biotechnological advances of family Apocynaceae: A review. Phytotherapy research, 32 (7), 1181-1210. https://doi.org/10.1002/ptr.6066

Borges, L. P., \& Amorim, V. A. (2020). Metabólitos secundários de plantas. Revista Agrotecnologia, 11 (1), 54-67. https://www.revista.ueg.br/index.php/agrotecnologia/article/view/9705

Cepleanu, F., Hamburger, M. O., Sordat, B., Msonthi, J. D., Gupta, M. P., Saadou, M., \& Hostettmann, K. (1994). Screening of tropical medicinal plants for molluscicidal, larvicidal, fungicidal and cytotoxic activities and brine shrimp toxicity. International Journal of Pharmacognosy, 32 (3), $294-307$. https://doi.org/10.3109/13880209409083007

Colombo, R. C., Favetta, V., Yamamoto, L. Y., Alves, G. A. C., Abati, J., Takahashi, L. S. A., \& Faria, R. T. (2015). Biometric description of fruits and seeds, germination and imbibition pattern of desert rose [Adenium obesum (Forssk.), Roem. \& Schult.] Journal of Seed Science, 37 (4), 206-213. https://doi.org/10.1590/2317-1545v37n4152811

Dimmitt, M. A., \& Hanson, C. (1991). The genus Adenium in cultivation. Part 1: A. obesum and A. multiflorum. Cactus and Succulent Journal, 63 (5), 223225. https://adenium.tucsoncactus.org/part1.html

Fabricant, D. S., \& Farnsworth, N. R. (2001). The value of plants used in traditional medicine for drug discovery. Environmental Health Perspectives, 109 (suppl 1), 69-75. https://dx.doi.org/10.1289\%2Fehp.01109s169

Gonçalves, K. G., \& Pasa, M. C. (2015). A etnobotânica e as plantas medicinais na Comunidade Sucuri, Cuiabá, MT, Brasil. Interações, 16 (2), 245-256. https://doi.org/10.1590/1518-70122015201 
Hoffmann, J. J., \& Cole, J. R. (1977). Phytochemical investigation of Adenium obesum Forskal (Apocynaceae): isolation and identification of cytotoxic agents. Journal of pharmaceutical sciences, 66 (9), 1336-1338. https://doi.org/10.1002/jps.2600660935

Hossain, M. A., Al-Mijizy, Z. H., Al-Rashdi, K. K., Weli, A. M., \& Al-Riyami, Q. (2013). Effect of temperature and extraction process on antioxidant activity of various leaves crude extracts of Thymus vulgaris. Journal Coastal Life Medicine. 1 (2), 118-122. http://dx.doi.org/10.12980/JCLM.1.2013C706

Hossain, M. A., Al-Abri, T. H. A., Al-Musalami, A. H. S., Akhtar, M. S., \& Said, S. (2014). Evaluation of different extraction methods on antimicrobial potency of Adenium obesum stem against food borne pathogenic bacterial strains in Oman. Asian Pacific Journal of Tropical Disease, 4 (Suppl 2), S985-S989. http://dx.doi.org/10.1016/S2222-1808(14)60770-2

Hossain, M. A., Akhtar, M. S., Said, S., \& Al-Abri, T. H. A. (2017). Two new flavonoids from Adenium obesum grown in Oman. Journal of King Saud University-Science, 29 (1), 62-69. http://dx.doi.org/10.1016/j.jksus.2016.04.004

Hossain, M. A. (2018). A review on Adenium obesum: A potential endemic medicinal plant in Oman. Beni-Suef University journal of basic and applied sciences, 7 (4), 559-563. https://doi.org/10.1016/j.bjbas.2018.06.008

Ibrahim, M. A., Mohammed, A., Isah, M. B., \& Aliyu, A. B. (2014). Anti-trypanosomal activity of African medicinal plants: A review update. Journal of Ethnopharmacology, 154(1), 26-54. https://doi.org/10.1016/j.jep.2014.04.012

Igbinosa, O. O., Igbinosa, E. O., \& Aiyegoro, O. A. (2009). Antimicrobial activity and phytochemical screening of stem bark extracts from Jatropha curcas (Linn). African journal of pharmacy and pharmacology, 32 (2), http://citeseerx.ist.psu.edu/viewdoc/download?doi=10.1.1.561.1288\&rep=rep1\&type=pdf

Kinghorn, A. D., \& Seo, E.K. (1996). Plants as Sources of Drugs. In: Agricultural Materials as Renewable Resources. Nonfood and Industrial Applications. Fuller, G., Mckeon, T. A. \& Bills, D. D. (ed.) ACS Symposium Series 647. Washington, DC. https://doi.org/10.1021/bk-1996-0647.ch012

Kiyohara, H., Ichino, C., Kawamura, Y., Nagai, T., Sato, N., Yamada, H., Salama, M. M., \& Sattar, E. A. (2012). In vitro anti-influenza virus activity of a cardiotonic glycoside from Adenium obesum (Forssk.). Phytomedicine, 19 (2), 111-114. https://doi.org/10.1016/j.phymed.2011.07.004

Limones, V., Baas, F. M., \& Borges I. (2018). La rosa del desierto (Adenium obesum): de exótica flor de ornato a interesante fuente de compuestos bioactivos. Desde el Herbario CICY, 10, 128-131. https://www.cicy.mx/Documentos/CICY/Desde_Herbario/2018/2018-06-14-VLimones-FBaas-IBorges-La-rosa-deldesierto.pdf

Mendes, K. D. S., Silveira, R. C. C. P., \& Galvão, C. M. (2008). Revisão integrativa: método de pesquisa para a incorporação de evidências na saúde e na enfermagem. Texto Contexto Enferm., 17 (4), 758-764. https://doi.org/10.1590/S0104-07072008000400018

McLaughlin, J., \& Garofalo, J. (2002). Desert Rose (Adenium obesum). Fact sheet 71. [Internet] Cooperative Extension Service, Miami-Dade County/University of Florida, Homestead, United States. http://miami-dade.ifas.ufl.edu/pdfs/ornamental/ornamental_publications/desert-rose.PDF.

Mgbojikwe, L. O., \& Okoye, Z. S. C. (2001). Acaricidal efficacy of aqueous stem bark extract of Adenium obesum on various life stages of cattle ticks. Nigerian Journal of Experimental and Applied Biology, 2 (1), 39-43.

Nagai, T., Moriguchi, R., Suzuki, Y., Tomimori, T., \& Yamada, H. (1995). Mode of action of the anti-influenza virus activity of plant flavonoid, 5,7,4'trihydroxy-8-methoxyflavone, from the roots of Scutellaria baicalensis. Antiviral Research, 26 (1), 11-25. https://doi.org/10.1016/0166-3542(94)00062-d

Nakamura, M., Ishibashi, M., Okuyama, E., Koyano, T., Kowithayakorn, T., Hayashi, M., Komiyama, K. (2000). Cytotoxic pregnanes from leaves of Adenium obesum. Natural medicines, 54 (3), 158-159.

Orozco, A. Z., \& Gonzalez, A. C. (2021). Bases para la mejora genética en la rosa del desierto (Adenium obesum). Repertorio Científico, 24 (1), $43-56$. https://doi.org/10.22458/rc.v24i1.3369

Oyen, L. P. A. (2008). Adenium obesum (Forssk.) Roem. \& Schult. In: Schmelzer, G.H., Gurib-Fakim, A., (Eds.), Plant Resources of Tropical Africa, Backhuys. Wageningen. Medicinal Plants 1, 11(1). https://edepot.wur.nl/417238

Paul, D., Biswas, K., \& Sinha, S. N. (2015). Biological activities of Adenium obesum (Forssk.) Roem. \& Schult.: a concise review. Malaya Journal of Biosciences, 2 (4), 214-221. https://www.researchgate.net/profile/Dipak-Paul2/publication/301847549_Biological_Activities_of_Adenium_obesum_Forssk_Roem_Schult_A_Concise_Review/links/572a0dd508ae2efbfdbc15d8/Biologic al-Activities-of-Adenium-obesum-Forssk-Roem-Schult-A-Concise-Review.pdf

Pengelly, A. (2021). The constituents of medicinal plants. Cabi publishing. https://www.cabi.org/bookshop/book/9781789243079/

Ramawat, K. G., \& Merillon, J. M. (2010). Bioactive molecules and medicinal plants. Heidelberg: Springer. https://link.springer.com/book/10.1007/978-3$540-74603-4$

Romahn, V. (2012). Enciclopédia ilustrada das plantas \& flores: suculentas, samambaias e aquáticas. São Paulo: Editora Europa, 144 p.

Silva, M.G.P., \& Silva, M.M.P. (2018). Avaliação do uso de fitoterápicos em distúrbios psiquiátricos. Revista de Atenção à Saúde, 16 (56), 77-82. DOI: https://doi.org/10.13037/ras.vol16n56.4951.

Silveira, M.P.C. (2016). Avaliação dos Parâmetros Ecofisiológicos e de Crescimento em Rosa do deserto sob restrição hídrica associada ao filme de partícula de $\mathrm{Caco}_{3}$. Dissertação (Mestrado em Agricultura e Biodiverssidade)- Universidade Federal de Sergipe, São Cristovão. https://ri.ufs.br/bitstream/riufs/3023/1/MARIA_PRISCILLA_CELESTINO_SILVEIRA.pdf

Souza, M.T., Silva, M.D., \& Carvalho, R. (2008). Revisão integrativa: o que é e como fazer. Einstein, 8 (1), 102-106. DOI: https://doi.org/10.1590/s167945082010rw1134. 
Research, Society and Development, v. 11, n. 2, e28211225843, 2022

(CC BY 4.0) | ISSN 2525-3409 | DOI: http://dx.doi.org/10.33448/rsd-v11i2.25843

Springfield, E. P., Eagles, P. K. F., \& Scott, G. (2005). Quality assessment of South African herbal medicines by means of HPLC fingerprinting. Journal of ethnopharmacology, 101 (1-3), 75-83. https://doi.org/10.1016/j.jep.2005.03.012

Tijjani, A., Ndukwe, I. G., \& Ayo, R. G. (2011). Studies on antibacterial activity of Adenium obesum (Apocynaceae) stembark. Continental Journal of Microbiology, 5 (1), 12-17. http://wiloludjournal.com/.../12-17.pdf

Toledo, M. M. (2008). A vulnerabilidade do adolescente ao HIV/ AIDS. Revisão integrativa. Dissertação (Mestrado em Enfermagem) -Universidade de São Paulo, São Paulo. teses.usp.br/teses/disponiveis/7/7137/tde-07072008-093333/publico/Melina_Mafra.pdf

Varella, T. L., Silva, G. M., Cruz, K. Z. C. M., Mikovski, A. I., Nunes, J. R. S., Carvalho, I. F., \& Silva, M. L. (2019). In vitro germination of desert rose varieties. Ornamental Horticulture, 21 (2), 227-234. https://doi.org/10.14295/aohl.v21i2.676

Versiani, M. A., Ahmed, S. K., Ikram, A., Ali, S. T., Yasmeen, K., \& Faizi, S. (2014). Chemical constituents and biological activities of Adenium obesum (Forsk.) Roem. et Schult. Chemistry \& biodiversity, 11 (2), 171-180. https://doi.org/10.1002/cbdv.201200254

Zu, M., Yang, F., Zhou, W., Liu, A., Du, G., \& Zheng, L. (2012). In vitro anti-influenza virus and anti-inflammatory activities of the aflavin derivatives. Antiviral Research, 94 (3), 217-224. https://doi.org/10.1016/j.antiviral.2012.04.001 